\title{
DNA methylation profiles of gastric carcinoma characterized by quantitative DNA methylation analysis
}

\author{
Gyeong Hoon Kang ${ }^{1}$, Sun Lee ${ }^{2}$, Nam-Yun Cho' ${ }^{1}$, Tasha Gandamihardja, ${ }^{3,4}$, Tiffany I Long ${ }^{3,4}$, \\ Daniel J Weisenberger ${ }^{3,4}$, Mihaela Campan ${ }^{3,4}$ and Peter W Laird ${ }^{3,4}$
}

Transcriptional silencing by $\mathrm{CpG}$ island hypermethylation is a potential mechanism for the inactivation of tumor-related genes. Virtually, all types of human cancers show CpG island hypermethylation, and gastric carcinoma (GC) is one of the tumors with a high frequency of aberrant $\mathrm{CpG}$ island hypermethylation. In this study, we prescreened DNA methylation of $170 \mathrm{CpG}$ island loci in a training set of 8 paired GC and GC-associated non-neoplastic mucosae (GCN) using MethyLight technology and selected 27 DNA methylation markers showing higher methylation frequency or level in GC than in GCN. These markers were then analyzed in a tester set of 25 paired GC and GCN and 27 chronic gastritis (CG) from non-cancer patients to generate their DNA methylation profiles. We identified 17 novel methylation markers in GC, including SFRP4, SEZ6L, TWIST1, BCL2, KL, TERT, SCGB3A1, IGF2, GRIN2B, SFRP5, DLEC1, HOXA1, CYP1B1, SMAD9, MT1G, $N R 3 C 1$, and HOXA10. Of the 27 selected CpG island loci, 23 were methylated in GC, GCN, and CG and the remainder four loci (DLEC1, CHFR, CYP1B1, and NR3C1) were only methylated in GC. We found that the number of methylated loci was significantly higher in GC than in GCN or CG and that Helicobacter pylori infection was strongly associated with aberrant CpG island hypermethylation in CG. Hypermethylation was more prevalent in Epstein-Barr virus (EBV)-positive GC than in EBV-negative GC and in diffuse-type GC than in intestinal-type GC. Through our large-scale screening of $170 \mathrm{CpG}$ island loci, we found 17 new DNA methylation markers of GC, which may serve as useful markers that may identify a distinct subset of GC.

Laboratory Investigation (2008) 88, 161-170; doi:10.1038/labinvest.3700707; published online 24 December 2007

KEYWORDS: CpG islands; DNA methylation; Epstein-Barr virus; gastric cancer; Helicobacter pylori

CpG islands are DNA segments, at least $0.5 \mathrm{~kb}$ in size, rich in $\mathrm{G}: \mathrm{C}$ and $\mathrm{CpG}$ content, and often located in the promoter or $5^{\prime}$-exon sequences of genes. ${ }^{1}$ Promoter $\mathrm{CpG}$ islands have traditionally been thought to be unmethylated in normal cells, with the exception of those on the inactive $\mathrm{X}$ chromosome and those associated with imprinted genes. Although the cause is unclear, promoter $\mathrm{CpG}$ island hypermethylation can occur in association with cancer development or aging. Promoter $\mathrm{CpG}$ island hypermethylation is closely associated with gene inactivation and thought to act as an alternative to genetic change for inactivation of tumor suppressor genes in human cancers. Virtually, all types of human cancer display promoter $\mathrm{CpG}$ island hypermethylation, although there are variations in the prevalence of $\mathrm{CpG}$ island hypermethylation among tumor types. ${ }^{2,3}$ In addition to its potential role in gene inactivation in human cancers, $\mathrm{CpG}$ island hypermethylation is now gaining attention as a molecular marker for tumor detection and prediction of development or progression of cancer. DNA methylation markers are actively being investigated for their utility as biomarkers to detect human cancers in blood, secretions, or exfoliated cytology specimens and to predict the risk of the progression or development of cancer. ${ }^{4,5}$

Gastric carcinoma (GC) is the most common cancer in eastern Asia and the third most frequent cancer across the world. ${ }^{6}$ The stomach is one of the organs where aberrant $\mathrm{CpG}$ island hypermethylation occurs frequently during cancer development or aging. ${ }^{7}$ To date, about 87 genes have been characterized to be inactivated by hypermethylation of their promoter $\mathrm{CpG}$ islands in GC. ${ }^{8}$ Considering the fact that

\footnotetext{
${ }^{1}$ Department of Pathology, Cancer Research Institute, Seoul National University College of Medicine, Seoul, Korea; ${ }^{2}$ Department of Pathology, Kyung Hee University College of Medicine, Seoul, Korea; ${ }^{3}$ Department of Surgery, Keck School of Medicine, Norris Comprehensive Cancer Center, University of Southern California, Los Angeles, CA, USA and ${ }^{4}$ Department of Biochemistry and Molecular Biology, Keck School of Medicine, Norris Comprehensive Cancer Center, University of Southern California, Los Angeles, CA, USA

Correspondence: Professor GH Kang, MD, Department of Pathology, Seoul National University College of Medicine and Cancer Research Institute, 28 Yongon-dong, Chongno-gu, Seoul 110-744, Korea. E-mail: ghkang@snu.ac.kr
} 
more than $40 \%$ of human genes contain promoters within CpG islands ${ }^{9-11}$ and the number of the genes characterized as hypermethylated in GCs is very limited, the vast majority of genes with $\mathrm{CpG}$ islands remain to be tested as to their DNA methylation status in GC.

Methylation-specific polymerase chain reaction (MSP) is a highly sensitive method and can detect one methylated allele in 10000 unmethylated alleles. ${ }^{12}$ However, MSP does not provide quantitative information on the proportion of the methylated alleles among total alleles. Combined bisulfite restriction analysis (COBRA) is a quantitative method detecting methylated alleles but less sensitive than MSP. Furthermore, it cannot be applied to all $\mathrm{CpG}$ island loci because of its dependence upon the limited number of restriction enzymes available that recognize CG sites in bisulfite-converted sequences. Most of the studies investigating $\mathrm{CpG}$ island hypermethylation in GCs used MSP, and the methylation frequencies of the specific CpG islands loci in GCs varied among the studies. Although the discrepancies might be related to ethnic differences of the study populations (materials), it is also likely that some of the variability in observed methylation frequency of specific $\mathrm{CpG}$ island loci could be caused by the differences in the number of the cycles of polymerase chain reaction (PCR), the amount of template DNA, the PCR mixture conditions, or the specific target $\mathrm{CpG}$ sites of the $\mathrm{CpG}$ island locus. The quantitative, real-time PCR-based MethyLight assay can overcome issues related to PCR cycling and can sensitively detect DNA methylation in a small amount of template DNA. MethyLight assay has been evaluated and validated for its precision and performance characteristics. ${ }^{13,14}$

In the present study, we identified DNA methylation markers that can differentiate GC from non-neoplastic gastric mucosae in terms of the methylation frequency or methylation level (the proportion of the methylated alleles among the total alleles, including methylated and unmethylated alleles). After prescreening of $170 \mathrm{CpG}$ island loci against a training set (8 paired GC and the associated nonneoplastic stomach tissues), we selected and analyzed $27 \mathrm{CpG}$ island loci for their methylation status in a tester set of GCs $(n=25)$, paired GC-associated non-neoplastic mucosae $(\mathrm{GCN})$, and chronic gastritis (CG) samples from patients without GC $(n=27)$ using MethyLight. The results of DNA methylation were analyzed in relation to clinicopathologic factors, including histologic type, microsatellite instability (MSI) status, and the presence of Helicobacter pylori (HP) or Epstein-Barr virus (EBV).

\section{MATERIALS AND METHODS \\ Patients}

Formalin-fixed, paraffin-embedded archival tissues from 33 GC patients and 27 CG patients were retrieved from the file of the Department of Pathology, Seoul National University Hospital (Seoul, Korea). The age of cancer patients ranged 36-81 years (mean: 62.4 years; median: 63 years) and male to female ratio was 4:1. The age of CG patients ranged 41-68 years (mean: 53.3 years; median: 53 years) and male to female ratio was 19:10. This study was approved by the Institutional Review Board.

\section{DNA Extraction and Bisulfite Modification}

Ten sections of $10 \mu \mathrm{m}$ thickness of paraffin-embedded tissues were used for DNA extraction. The paraffin was removed from the tissue by rinsing in xylene and genomic DNA was isolated using a QIAamp tissue kit (Qiagen, Valencia, CA, USA). Sodium bisulfite conversion of genomic DNA was performed as follows. Briefly, $2 \mu \mathrm{g}$ of genomic DNA was denatured at $100^{\circ} \mathrm{C}$ for $10 \mathrm{~min}$, followed by incubation in $0.3 \mathrm{M} \mathrm{NaOH}$ at $42^{\circ} \mathrm{C}$ for $20 \mathrm{~min}$. Bisulfite conversion was performed with $2.5 \mathrm{M}$ sodium metabisulfite solution at $50^{\circ} \mathrm{C}$ for $16 \mathrm{~h}$ in the dark. DNA was purified from the bisulfite solution using QIAamp Viral RNA Mini Kit (Qiagen) according to the manufacturer's recommendations, except that each sample was loaded two times onto each spin column. After the entire sample was loaded and the spin column washed, the sample was eluted twice in $40 \mu \mathrm{l}$ elution buffer. The $80 \mu \mathrm{l}$ sample was then desulfonated by incubation in $0.077 \mathrm{M} \mathrm{NaOH}$ solution at room temperature for $15 \mathrm{~min}$. After neutralization with $1 \mathrm{M} \mathrm{HCl}$, DNA was purified a second time using the QIAamp Viral RNA Mini Kit (Qiagen). The eluted sample $(80 \mu \mathrm{l})$ was then diluted accordingly for use in MethyLight analysis.

\section{DNA Methylation Analysis}

DNA methylation analyses were performed using MethyLight. We screened 170 DNA MethyLight reactions to identify those that are cancer-specifically methylated in GCs. Most of these MethyLight reactions had been developed for other purposes, including studies of esophageal cancer, lung cancer, pancreatic cancer, ovarian cancer, brain cancer, and neurodegenerative disorders. From this prescreen, we selected 27 MethyLight reactions for subsequent DNA methylation analyses. These MethyLight reaction primers, probes, and information are shown in Supplementary Table 1. MethyLight PCR was performed in a $30 \mu \mathrm{l}$ reaction volume with $200 \mathrm{mM}$ dNTPs, $0.3 \mu \mathrm{M}$ forward and reverse PCR primers, $0.1 \mu \mathrm{M}$ probe, $3.5 \mathrm{mM} \mathrm{MgCl}_{2}, \quad 0.01 \%$ Tween-20, $0.05 \%$ gelatin and $0.1 \mathrm{U}$ of Taq polymerase using the following PCR program: $95^{\circ} \mathrm{C}$ for $10 \mathrm{~min}$, then 50 cycles of $95^{\circ} \mathrm{C}$ for $15 \mathrm{~s}$ followed by $60^{\circ} \mathrm{C}$ for $1 \mathrm{~min}$. The samples in 96-well plates were analyzed on an Opticon DNA Engine Continuous Fluorescence Detector (MJ Research/Bio-Rad, Hercules, CA, USA). Each MethyLight reaction signal was compared to two control reactions, $A L U$ and $C O L 2 A 1$, to normalize for input bisulfite-converted DNA levels. The COL2A1 reaction and the $A L U$ reaction were described previously. ${ }^{15,16}$ Both COL2A1 and $A L U$ reactions are specific for bisulfite-converted DNA but are not DNA methylation specific. The specificity of the reactions for methylated DNA was confirmed separately using M.SssI (New England Biolabs 
Inc., Beverly, MA, USA)-treated human peripheral blood lymphocyte DNA (Promega Co., Madison, WI, USA). The percentage of fully methylated alleles (percentage of methylated reference $(\mathrm{PMR})^{4}$ ) at each locus was calculated by dividing the GENE:ALU ratio of a sample by the GENE:ALU ratio of the M.SssI-treated human genomic DNA sample and multiplying by 100 . The same calculation was performed for each sample using the COL2A1 control reaction, and the final PMR values were obtained by averaging the PMR values based on the individual $A L U$ and COL2A1 control reactions. Since the PMR value is calculated as a ratio of the signal between the sample and in vitro M.SssI-treated reference sample, there may be instances in which the PMR value is greater than 100 if there was incomplete methylation of the reference sample. PMR calculations have also been described extensively. ${ }^{4,13,16}$ There is no standard cutoff value to classify methylated and unmethylated loci using DNA methylation analyses, so we considered a CpG island locus methylated if the PMR value was $>4$. Our rationale is that samples with PMR values less than 4 are not substantially methylated, and therefore may not serve well as DNA methylation markers in GC, and the PMR cutoff value of 4 has been validated by previous studies based on the distributions of PMR values in the tested CpG island loci and correlation of protein expression loss with methylation positivity in CDKN2A, MLH1, and MGMT determined by the PMR cutoff of $4 .{ }^{14,17}$

\section{Microsatellite Instability Analysis}

The MSI status of each tumor and paired normal mucosa sample was determined based on an examination of five microsatellite markers (D2S123, D5S346, D17S250, BAT25, and BAT26). Either forward or reverse primer for each marker was labeled with fluorescence, and PCR products were electrophoresed and analyzed by ABI 3730 (Applied Biosystems, Foster City, CA, USA). We classified tumors with instability at two or more microsatellite loci as MSI-positive.

\section{Epstein-Barr Virus-Encoded RNA In Situ Hybridization}

The EBV RNA in situ hybridization was performed using a fluorescein-conjugated peptide nucleic acid probe complimentary to a portion of the small EBV-encoded RNAs (EBERs) 1 and 2. Sections ( $5 \mu \mathrm{m}$ thick) on slides coated with poly-L-lysine were routinely deparaffinized, dehydrated, and predigested with $3 \mu \mathrm{g} / \mathrm{ml}$ proteinase $\mathrm{K}$, and then hybridized for $2 \mathrm{~h}$ at $37^{\circ} \mathrm{C}$. Anti-fluorescein antibody conjugated to alkaline phosphatase was used with an NBT/BCIP kit (Dako, Copenhagen, Denmark) to detect the EBER signals. Counterstaining was performed with Mayer's hematoxylin.

\section{Assays For $\boldsymbol{H}$. pylori Infection}

The presence of HP infection was determined by light microscopic examination of Giemsa-stained histologic slides, PCR for HP 16S rDNA, or rapid urease test (CLO test; Delta West, Bentley, WA, Australia). Cases that showed positivity in any of the three assays were regarded as HP-positive. PCR for HP 16S rDNA was performed as previously described. ${ }^{18}$

\section{5-Aza-2'-deoxycytidine and Trichostatin A treatments}

Cells were seeded at a density of $3 \times 10^{5}$ cells $/ 10 \mathrm{~cm}$ dish on day 0 and treated daily with $1 \mu \mathrm{M} 5$-aza- $2^{\prime}$-deoxycytidine (5-Aza-CdR) (Sigma Chemical Co., St Louis, MO, USA) for $72 \mathrm{~h}$. We also treated cells with $150 \mathrm{nM}$ Trichostatin A (Sigma Chemical Co.) alone for $24 \mathrm{~h}$ or in combination of $1 \mu \mathrm{M}$ 5-Aza-CdR (72 h) plus $150 \mathrm{nM}$ Trichostatin A (24h). After the treatments, the cells were harvested and RNA was extracted for reverse transcription-PCR (RT-PCR).

\section{RT-PCR}

cDNA was synthesized from $1 \mu \mathrm{g}$ of total RNA treated with DNase I (Ambion, Austin, TX, USA) with a Superscript II kit (Invitrogen, Carlsbad, CA, USA). The quantity of mRNA was normalized to that of GAPDH. The primers and PCR conditions are shown in Supplementary Table 2. Real-time RT-PCR was carried out using Power SYBR Green PCR Master Mix (Applied Biosystems) in an iCycler Optical Module (Bio-Rad).

\section{Statistical Analysis}

The Student's $t$-test was used to compare the number of $\mathrm{CpG}$ island loci methylated or methylation index (MI) in GC, GC-paired normal mucosa, and normal mucosa from non-cancer patients. To compare means between paired samples, paired $t$-test was used. SPSS software was used throughout (SPSS for Windows Release, Ver.11.0; SPSS Inc., Chicago, IL, USA). $P$-values of $<0.05$ were accepted as being statistically significant.

\section{RESULTS}

\section{Selection of 27 CpG Islands Loci from the MethyLight} Assay of $170 \mathrm{CpG}$ Islands Loci in a Training Set

DNA methylation markers $(n=170)$ were prescreened for a training set of eight normal tumor pairs of GCN and GC samples to determine those markers that differentiate between GC and GCN on the combined aspects of methylation frequencies and DNA methylation levels. We first removed 32 markers that exhibited a maximum PMR value $<0.01$ in GCN and GC samples, and 38 markers displaying a minimal PMR value $>4$ only in GCN and GC samples. From the remaining 100 methylation markers, 27 informative markers were selected by the following criteria: first, we determined the mean PMR values of each marker for both normal (GCN) and tumor (GC) samples. We next determined the ratio of mean tumor and normal PMR values using the following formula: [Mean $\mathrm{PMR}_{\text {tumor }} /$ (Mean $\left.\mathrm{PMR}_{\text {normal }}+1\right)$ ]. Second, we determined the number of cases in which the PMR value of each methylation marker was greater than 0 for the GC samples and paired GCN samples. GC/GCN frequencies were determined using the formula $\left[\right.$ Number $_{\text {tumor }} /\left(\right.$ Number $\left.\left._{\text {normal }}+1\right)\right]$. Finally, we 
determined the product of the PMR values and frequencies $\left[\left(\right.\right.$ mean $\mathrm{PMR}_{\text {tumor }} /\left(\right.$ Mean $\left.\left.\mathrm{PMR}_{\text {normal }}+1\right)\right) \times\left(\right.$ Number $_{\text {tumor }} /$ $\left(\right.$ Number $\left.\left.\left._{\text {normal }}+1\right)\right)\right]$ among the GC and GCN samples and ranked these in descending order. The best 27 markers were selected and examined on a tester set of 25 paired GCN and GC samples and 27 CG samples from non-cancer patients. The oligonucleotide sequences of the primers and probes of the 27 methylation markers are listed in Supplementary Table 1.

MethyLight Analysis of 27 CpG Islands Loci in a Tester Set of Gastric Carcinomas, Paired Gastric CarcinomaAssociated Non-Neoplastic Mucosae, and Chronic Gastritis from Non-Cancer Patients

We performed MethyLight analysis of $27 \mathrm{CpG}$ island loci on GC and paired GCN, as well as CG from non-cancer patients, and we calculated PMR values for each sample and MethyLight reaction (Figure 1). We also calculated DNA methylation frequencies $(\mathrm{PMR}>4)$ for each sample. These values are summarized in Table 1. The methylation frequencies of 27 CpG island loci ranged from 24 to $96 \%$ in a tester set of GC samples. High methylation frequencies ( $>50 \%$ of GC samples) were evident for ITGA4, SFRP4, TWIST1, SEZ6L, BCL2, TERT, KL, RBP1, SCGB3A1, RUNX3, GRIN2B, SFRP5, $I G F 2$, and DLEC1 (in descending order of methylation frequency). CACNA1G, CHFR, HOXA1, CYP1B1, BDNF, CDKN2A, SMAD9, RARRES1, CDKN1C, and MT1G (in order of decreasing methylation frequency) showed an intermediate frequency of methylation (30-50\%), while NR3C1, TP73, and HOXA10 were methylated between 20 and $30 \%$ of GC samples.

When the PMR values of each CpG island locus were compared between GC and paired GCN samples, all loci except RARRES1, CDKN1C, MT1G, NR3C1, and HOXA10 were methylated in GC at levels significantly higher than those of the respective ones in GCN samples $(P<0.05$, paired $t$-test $)$. Although the differences of methylation levels were not significant, RARRES1, CDKN1C, NR3C1, and HOXA10 were methylated in $\mathrm{GC}$ at levels three times higher than those of GCN.

\section{RNA Expression Levels of 17 CpG Island Loci in Gastric Cancer Cell Lines}

To evaluate whether the DNA hypermethylation events are inversely correlative with gene expression, we treated eight gastric cancer cell lines with 5-Aza-CdR and or Trichostatin A. After the drug treatment(s), we harvested total RNA and evaluated the expression of 17 gene mRNAs by RT-PCR. The primers are described in Supplementary Table 2. We also obtained MethyLight-based DNA methylation measurements of the CpG islands associated with each gene (Figure $2 \mathrm{a}$ ). In general, there was an inverse correlation between MethyLight PMR value and the gene expression levels and many of the genes had upregulated expression after 5-Aza-CdR treatment (Figure $2 \mathrm{a}-\mathrm{c}$ ). These findings suggest that the DNA hypermethylation seen in the clinical GC cases may have a functional and biological relevance. However, there were some loci that did not show an increase in gene expression after drug treatments.

\section{Comparison of Methylation Levels or Frequencies between $\boldsymbol{H}$. pylori-Positive and -Negative Non-Neoplastic Gastric Mucosae}

We determined the status of HP infection using microscopic examination of Giemsa-stained histologic slides, PCR assays to amplify HP $16 \mathrm{~S}$ rDNA (Supplementary Figure 1), and CLO test. The cases were regarded as HP-positive when they showed positive findings in any of the above three tests. Of 27 CG samples from non-cancer subjects, 13 samples were HP-negative and 14 samples were HP-positive. The methylation levels of $10 \mathrm{CpG}$ island loci, including SEZ6L, CDKN1C, TWIST1, CDKN2A, RBP1, SFRP4, MT1G, SFRP5, RARRES1, and SMAD9, were significantly higher in HPpositive CG than those in HP-negative CG (Figure 3a). When the number of $\mathrm{CpG}$ island loci methylated was compared between HP-positive and HP-negative CGs, HP-positive CG exhibited significantly higher number of $\mathrm{CpG}$ island loci methylated than HP-negative CG (12.1 vs 5.7, $P=0.001$, Student's $t$-test) (Figure $3 \mathrm{~b}$ ).

In addition, we found that 22 of 25 (88\%) GCN samples from GC patients were found to be HP-positive, and the remaining three cases were HP-negative. The differences in the methylation levels of the examined $\mathrm{CpG}$ island loci between HP-positive and HP-negative GCN from GC patients displayed similar trend seen in the above comparison, although the limitation of the case number of HP-negative GCN cases did not give credit upon this comparison.

\section{The Distribution of CpG Island Hypermethylation in Normal Gastric Mucosae from Non-Cancer Patients, Normal Gastric Mucosae Associated with Gastric Carcinomas, and Gastric Carcinomas}

The number of $\mathrm{CpG}$ island loci methylated varied from 0 to 17 in CG samples from non-cancer patients (median: 9) and from 5 to 17 in GCN samples (median: 12) and from 2 to 27 loci in GC samples (median: 14). When the MI was defined as the number of methylated loci/the number of tested loci, the average MI was $0.33,0.43$, and 0.54 for CG, GCN, and GC, respectively. The differences in MI values between CG and GCN and between GCN and GC were near statistical significance $(P=0.049$, two-tailed $t$-test and $P=0.077$, twotailed paired sample test, respectively). However, after taking into consideration the fact that HP prevalence was higher in GCN than in CG, we performed comparison of MI between HP-positive CG and HP-positive GCN, which revealed no difference of MI between them (MI, 0.45 vs 0.44 ).

The Relationship between CpG Island Hypermethylation and Histologic Type of Gastric Carcinoma, Epstein-Barr Virus, or Microsatellite Instability

According to the Lauren's classification, ${ }^{19}$ there are two major histologic types of GC, intestinal and diffuse types. Of the GC 


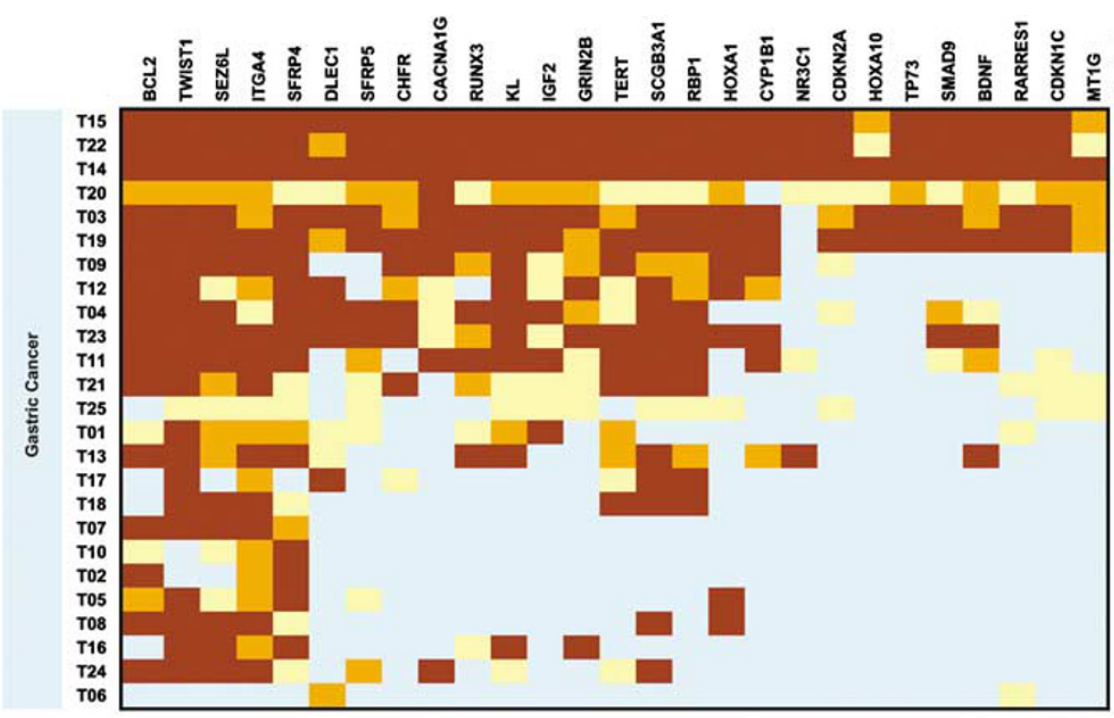

Figure 1 Methylation map of the PMR values obtained for each $\mathrm{CpG}$ island locus in gastric carcinoma (GC), GC-associated normal gastric mucosa, and normal gastric mucosa from non-cancer patients. Colored boxes represent four classes of methylation levels $(0<\mathrm{PMR}<4$ (light blue), $4 \leq \mathrm{PMR}<20$ (light yellow), $20 \leq \mathrm{PMR}<50$ (light orange), $\mathrm{PMR} \geq 50$ (brown)). EBV: +, EBV-positive GC; MSI: + , MSI-positive GC. Lauren's classification of histologic type: $D$, diffuse type; I, intestinal type.
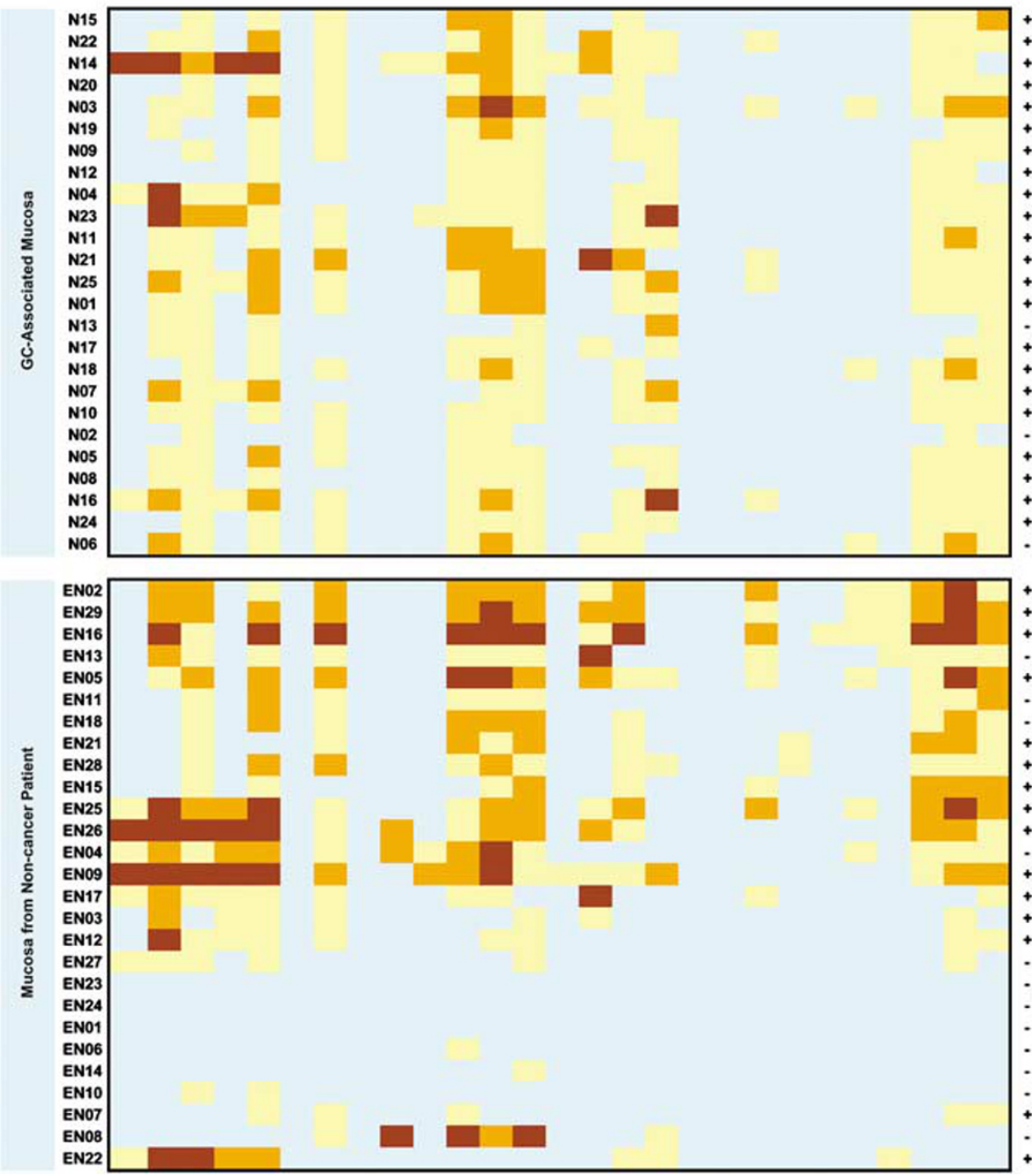

tester samples, 13 were of intestinal type and 12 were classified as diffuse type. Diffuse-type GCs showed higher MI than that of intestinal type $(0.33$ and 0.76 for intestinal type and diffuse type, respectively; $P<0.001$, two-tailed Student's $t$-test).
A close association has been known between CpG island hypermethylation and EBV positivity or MSI positivity in GCs. $^{20-23}$ To confirm the association, EBER in situ hybridization was performed for tissue array of 25 cases of 
Table 1 Methylation frequencies and PMR values of 27 analyzed genes in GC, GC-associated normal gastric mucosa, and normal gastric mucosa from non-cancer patients

\begin{tabular}{|c|c|c|c|c|c|c|}
\hline & \multicolumn{2}{|l|}{ GC } & \multicolumn{2}{|c|}{$\begin{array}{l}\text { GC-associated } \\
\text { gastric mucosa }\end{array}$} & \multicolumn{2}{|c|}{$\begin{array}{l}\text { Normal gastric } \\
\text { mucosa from non- } \\
\text { cancer patients }\end{array}$} \\
\hline & $\begin{array}{l}\text { Frequency } \\
\text { (\%) }\end{array}$ & PMR & $\begin{array}{l}\text { Frequency } \\
(\%)\end{array}$ & PMR & $\begin{array}{l}\text { Frequency } \\
(\%)\end{array}$ & PMR \\
\hline ITGA4 & 96 & 80.2 & 24 & 6.3 & 29.6 & 10.7 \\
\hline SFRP4 & 92 & 78.6 & 96 & 19.3 & 74.1 & 26.0 \\
\hline SEZ6L & 88 & 78.2 & 92 & 12.0 & 70.4 & 17.1 \\
\hline TWIST1 & 88 & 214.7 & 72 & 16.4 & 51.9 & 39.1 \\
\hline $\mathrm{BCL} 2$ & 80 & 97.3 & 12 & 4.5 & 25.9 & 10.7 \\
\hline $\mathrm{KL}$ & 68 & 105.3 & 92 & 13.2 & 63.0 & 22.2 \\
\hline TERT & 68 & 44.4 & 4 & 0.3 & 3.7 & 0.6 \\
\hline RBP1 & 64 & 58.1 & 80 & 8.7 & 44.4 & 8.8 \\
\hline SCGB3A1 & 64 & 152.6 & 24 & 9.0 & 37.0 & 11.9 \\
\hline IGF2 & 56 & 56.1 & 96 & 21.0 & 63.0 & 20.7 \\
\hline GRIN2B & 56 & 52.0 & 96 & 13.5 & 70.4 & 28.0 \\
\hline SFRP5 & 56 & 29.8 & 76 & 9.1 & 66.7 & 13.2 \\
\hline RUNX3 & 56 & 43.9 & 8 & 0.9 & 7.4 & 1.3 \\
\hline DLEC1 & 52 & 28.4 & 0 & 0.2 & 0.0 & 0.2 \\
\hline HOXA1 & 48 & 76.3 & 72 & 14.7 & 18.5 & 2.2 \\
\hline CHFR & 48 & 59.7 & 0 & 0.1 & 0.0 & 0.2 \\
\hline CACNA1G & 48 & 76.7 & 4 & 0.5 & 11.1 & 6.2 \\
\hline CYP1B1 & 40 & 68.1 & 0 & 0.1 & 0.0 & 0.0 \\
\hline BDNF & 40 & 31.5 & 0 & 0.5 & 18.5 & 1.8 \\
\hline RARRES1 & 36 & 25.2 & 84 & 7.6 & 51.9 & 14.3 \\
\hline CDKN1C & 36 & 43.7 & 96 & 14.5 & 66.7 & 24.3 \\
\hline CDKN2A & 36 & 18.1 & 20 & 1.9 & 29.6 & 6.0 \\
\hline SMAD9 & 36 & 33.3 & 12 & 1.4 & 22.2 & 2.4 \\
\hline MT1G & 32 & 13.1 & 88 & 9.4 & 63.0 & 13.0 \\
\hline NR3C1 & 24 & 22.2 & 0 & 0.0 & 0.0 & 0.0 \\
\hline TP73 & 24 & 33.8 & 0 & 0.1 & 3.7 & 0.3 \\
\hline HOXA10 & 24 & 10.4 & 0 & 1.5 & 7.4 & 1.2 \\
\hline
\end{tabular}

GC samples (Supplementary Figure 2) and MSI of BAT25, BAT26, D2S123, D5S346, and D17S250 was assessed for 25 cases of GC. GCs with MSI in two or more loci were regarded as MSI-positive. Six cases were positive for EBV and five cases were positive for MSI. However, there was no case that was positive for both EBV and MSI. The average MI values were 0.98, 0.48, and 0.37 for EBV-positive GCs, MSI-positive GCs, and GCs negative for EBV and MSI, respectively. The MI difference between EBV-positive GCs and EBV-negative GCs was statistically significant $(P<0.001$, two-tailed Student's $t$-test); however, we did not find a statistically significant difference between MSI-positive GCs and GCs negative for EBV and MSI $(P>0.05$, two-tailed Student's $t$-test).

\section{DISCUSSION}

Methylation in GC was revealed for the first time in this study for 17 of the 27 markers, including SFRP4, SEZ6L, TWIST1, BCL2, KL, TERT, SCGB3A1, IGF2, GRIN2B, SFRP5, DLEC1, HOXA1, CYP1B1, SMAD9, MT1G, NR3C1, and HOXA10. RNA expression levels of the 17 genes were analyzed in eight GC cell lines and correlated with the methylation status of the respective genes, suggesting that the DNA hypermethylation events in human GC are biologically important for gene function. A connection between DNA methylation and gene expression has been frequently demonstrated in the literature, however, not all CpG island hypermethylation show this correlation, as seen here as well. Many genes are not expressed in normal or tumor epithelium and their methylation in cancer is simply a marking of cancer, and has nothing to do with a functional or biological role. The co-authors of the present study recently published a report describing that there is a strong agreement between colorectal cancer-specific CpG island DNA methylation and their polycomb (PcG) occupancy in human embryonic stem cells. ${ }^{24}$ Gene methylation of several PcG-target genes, including NEUROG1, NEUROD1, and MYOD1, which are not expressed in the normal colonic epithelium, occurs frequently in colorectal cancer. The expression of muscle differentiation (MYOD1) and neuronal development genes (NEUROG1 and NEUROD1) is not needed in normal colonic mucosa. Similar genes exist in gastric and other human cancers.

These newly developed methylation markers showed methylation frequencies $\geq 24 \%$ in GC. The methylation frequencies of BCL2, TERT, DLEC1, CYP1B1, and NR3C1 in $\mathrm{GC}$ were three times higher than those of the respective gene in GCN or CG from non-cancer patients. The methylation frequency of HOXA10 in GC was two times higher than that in GCN or CG. The methylation frequencies of SFRP4, SEZ6L, TWIST1, KL, IGF2, GRIN2B, SFRP5, HOXA1, and SMAD9 were not different between GC and GCN or CG, although the methylation level of each gene was at least twice higher in GC than that of the respective gene in CG or GCN.

The number of methylated CpG island loci was higher in HP-positive CG than in HP-negative CG. This result is consistent with those of previous studies that have demonstrated the close association of HP infection with aberrant $\mathrm{CpG}$ island hypermethylation. ${ }^{25-27}$ We found that HP infection was a confounding factor in the comparison of methylation between CG and GCN and the analysis restricted to HP-positive cases did not show any difference in the number of methylated genes between CG and GCN. This was in contrast to the previous study of Waki et al, ${ }^{28}$ who demonstrated higher number of methylated $\mathrm{CpG}$ island loci in GCN than in CG using MSP. However, they did not analyze the association between $\mathrm{CpG}$ island hypermethylation and HP infection. Recent studies exhibited a marked difference of methylation levels between CG and GCN in HP-negative cases but no difference between them in HP-positive cases. $^{27,29}$ 
a

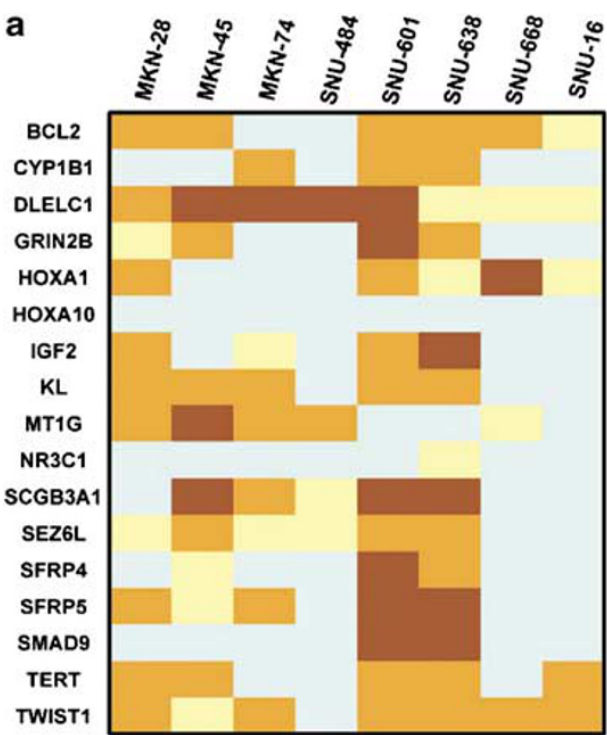

Figure 2 MethyLight analysis results of 17 genes in eight gastric cancer cell lines (a). Relative expression levels of 17 genes in eight gastric cancer cell lines (b and $\mathbf{c}$ ). Cells were treated with phosphate-buffered saline (PBS), 5-aza-2'-deoxycytidine (Aza), Trichostatin A (TSA), or combination of AZA plus TSA. The expression levels are displayed as the ratios between the individual genes and the endogenous control GAPDH and multiplied by a factor of 100. General inverse correlation between gene expression and $\mathrm{CpG}$ island hypermethylation was found in the vast majority of genes except for BCL2, IGF2, and TERT.
$4 \leq \mathrm{PMR}<20$

PMR $<4$

\section{b}
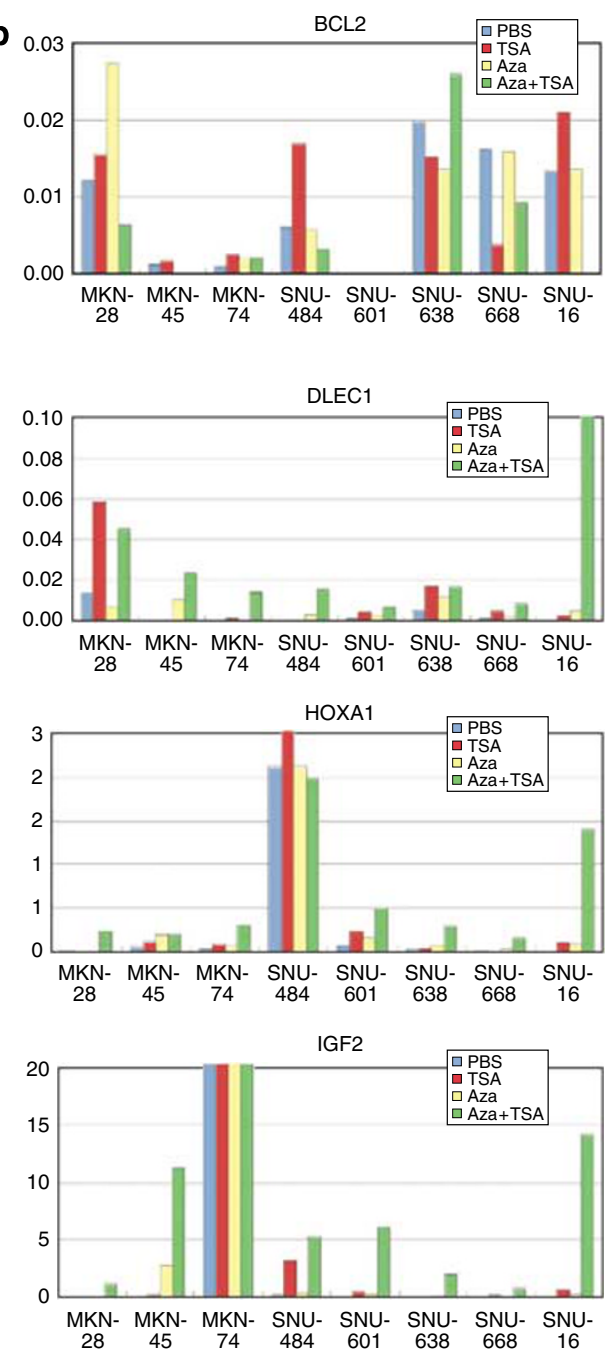
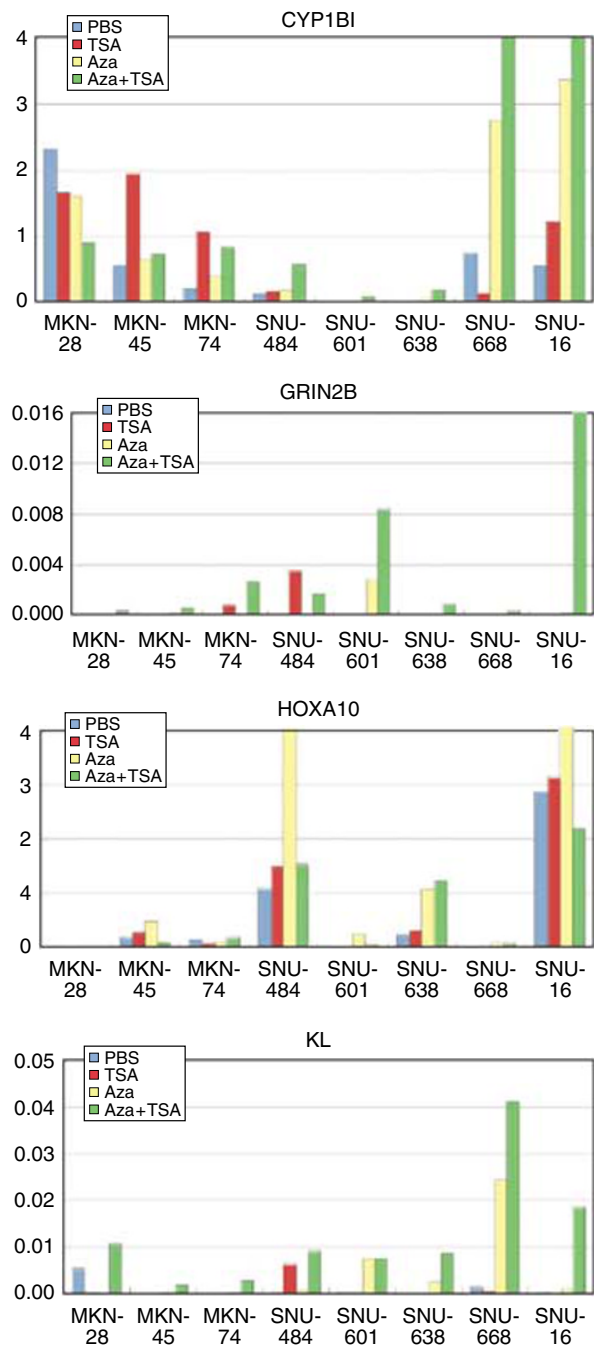

The CpG island locus of CACNA1G corresponds to MINT31, which was used as one of the reference CpG island loci to determine $\mathrm{CpG}$ island methylator phenotype of GCs or colorectal carcinomas. ${ }^{20,30,31}$ According to Toyota et al's ${ }^{30}$ study using COBRA, MINT31 was never methylated in GC-associated normal stomach samples. However, in our 

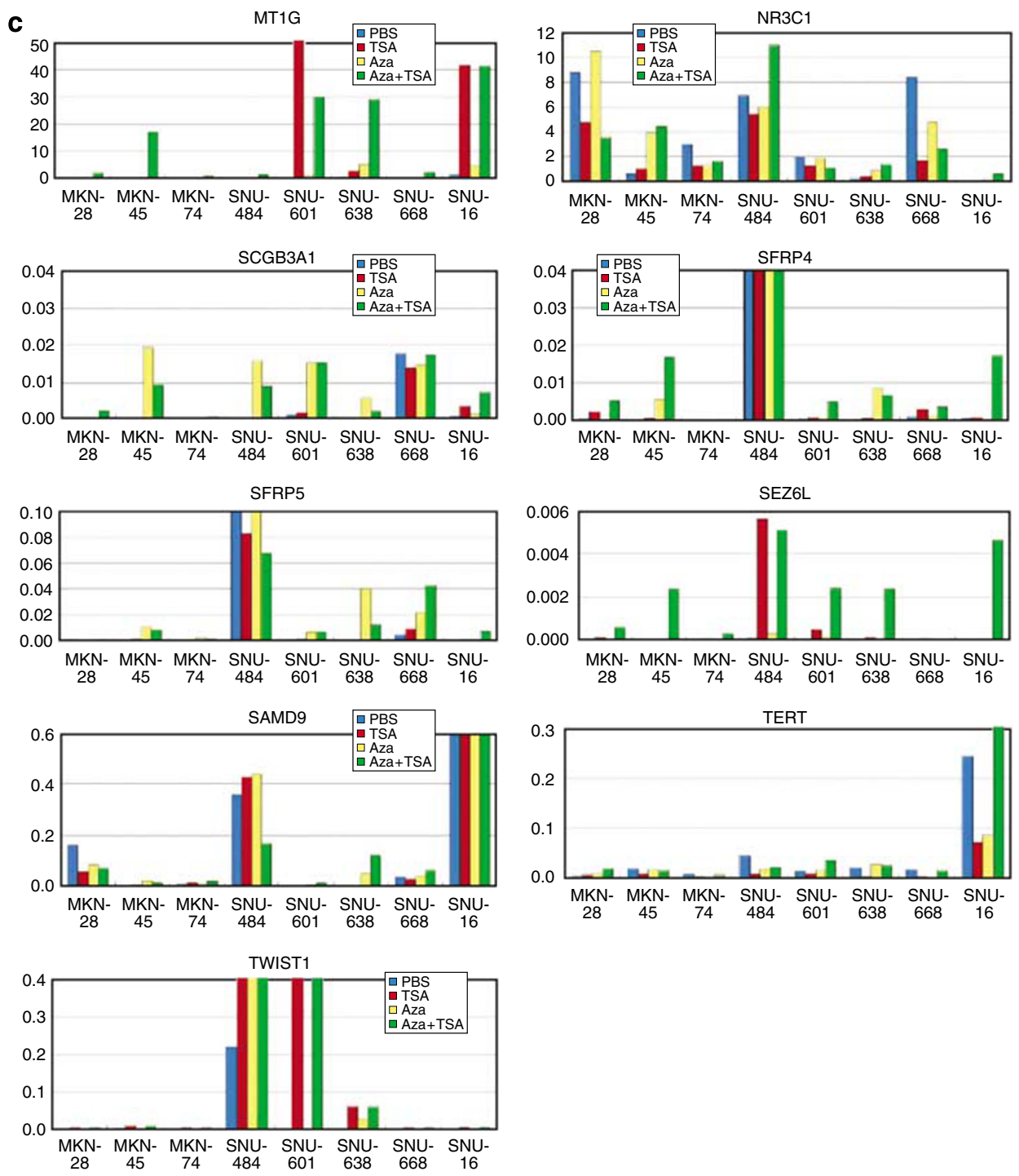

Figure 2 Continued.

study using MethyLight assay, CACNA1G was methylated in 3 of 27 CG samples from non-cancer patients and the mean PMR value of these 3 cases was 55. Considering the high PMR values of the three cases, it is likely that the same cases would be positive in the methylation analysis using COBRA for MINT31. Thus, the classification of MINT31 as a type $\mathrm{C}$ gene may need to be re-evaluated for gastric samples.

In previous studies, EBV-positive GCs and MSI-positive GCs have been demonstrated to contain a higher frequency of aberrant $\mathrm{CpG}$ island hypermethylation than GCs negative for both EBV and MSI. ${ }^{21-23,30,32,33}$ However, these previous studies used a small number of CpG island loci by MSP. In our study analyzing $27 \mathrm{CpG}$ island loci by MethyLight, which overcomes some variability problems of MSP, the average MI of EBV-positive GCs and MSI-positive GCs was 0.98 and 0.48 , respectively, whereas that of GCs negative for both was
0.37. Although a strong association was confirmed between $\mathrm{CpG}$ island hypermethylation and EBV, the association between CpG island hypermethylation and MSI was not found. This might be related to the small number of GCs studied in the present study. TP73 hypermethylation has been previously reported to be overrepresented in EBV-positive GCs (92.3\%, 12 of 13 EBV-positive GCs) but underrepresented in EBV-negative GCs $(5.3 \%, 2$ of 38 EBV-negative GCs). ${ }^{34}$ In the present study, TP73 hypermethylation was found in all the EBV-positive GCs but not found in EBV-negative GCs. Additionally, HOXA10 hypermethylation was also found in all the EBV-positive GCs but not found in EBV-negative GCs. These two DNA methylation markers are expected to serve as useful biomarkers to detect a distinct type of GC (EBV-positive GC).

GCs can be classified into two major histologic types: 'intestinal' and 'diffuse'. Intestinal-type GCs are associated 

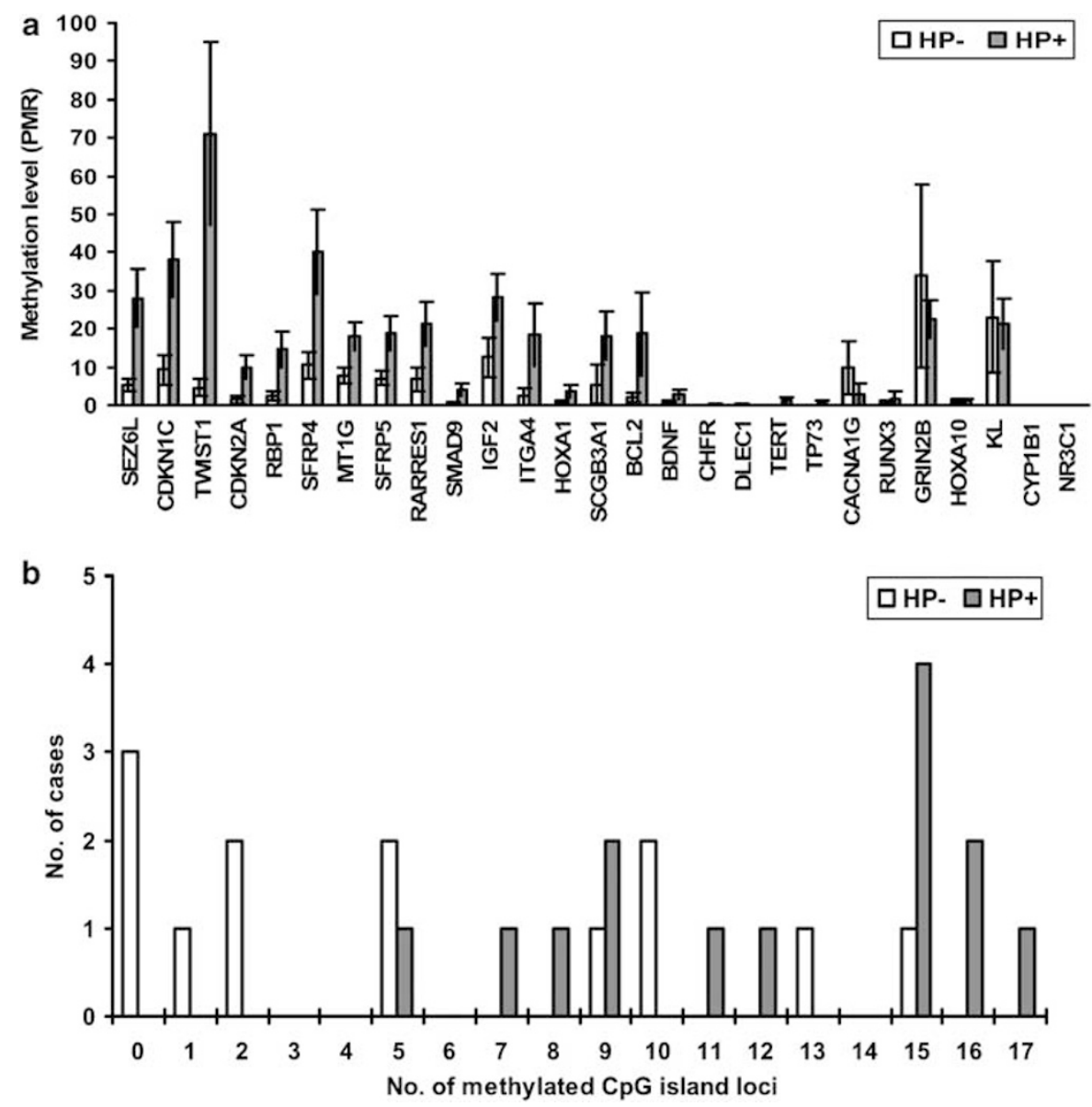

Figure 3 (a) The methylation levels of each of 27 CpG island loci were compared between HPpositive and HP-negative CG from non-cancer patients. Nine CpG island loci (from SEZ6L to SMD9) were methylated in HP-positive CG at significantly higher methylation levels compared with those of HP-negative CG. (b) The frequency of coincident number of gene hypermethylation was compared between HP-positive and HP-negative CG from non-cancer patients. with intestinal metaplasia, often preceded by sequential steps of precancerous changes, including atrophic gastritis, intestinal metaplasia, and dysplasia, whereas diffuse-type GCs arise de novo and originate from gastric mucosa proper. The pathogenesis and molecular alterations leading to these two types of GC are generally considered to differ. ${ }^{35-37}$ The difference in $\mathrm{CpG}$ island hypermethylation between intestinal and diffuse types has not been well characterized. Using different DNA methylation markers and different methodology, CpG island methylator phenotype was found to be associated with 'diffuse' histology in some reports ${ }^{33,38}$ but not in others. ${ }^{39,40}$ These controversial findings are most likely due to the use of differing DNA methylation marker panels as well as methods of analysis. In the present study, we analyzed 27 DNA methylation markers using the MethyLight technology and diffuse-type GC showed a significantly higher frequency of $\mathrm{CpG}$ island hypermethylation than intestinaltype GC, although the number of cases was limited. Considering that all the EBV-positive GCs were of diffuse type and comprised 50\% of diffuse-type GCs of the present study, the relationship between $\mathrm{CpG}$ island hypermethylation and diffuse type might depend on the proportion of EBV-positive GCs among the diffuse-type GCs. When
EBV-positive GCs were excluded from the diffuse-type GCs, still diffuse type showed higher MI than that of intestinal type (0.54 vs $0.33, P=0.054$, two-tailed Student's $t$-test).

In conclusion, we have generated DNA methylation profiles of 27 CGs from non-cancer patients and 25 paired GCs and GCNs and developed 17 new methylation markers for GC. We found that HP infection was strongly associated with aberrant $\mathrm{CpG}$ island hypermethylation in CG and the number of methylated $\mathrm{CpG}$ island loci was significantly higher in GC than in its associated GCN. CpG island hypermethylation was more prevalent in diffuse-type GC than in intestinal-type GC and EBV-positive GC showed methylation in more than $95 \%$ of the loci interrogated.

Supplementary Information accompanies the paper on the Laboratory Investigation website (http://www.laboratoryinvestigation.org)

\section{ACKNOWLEDGEMENT}

We thank Eun Joo Yoo for her technical support. This work was supported by the NIH Grant R01 CA001815 from the National Cancer Institute (to PWL) and the Korea Research Foundation Grant (MOEHRD) (KRF-2005-041E00081) (to GHK). 


\section{DISCLOSURE/DUALITY OF INTEREST}

Peter W Laird is a shareholder and Scientific Advisory Board Member of Epigenomics, AG, which has a commercial interest in DNA methylation markers. None of the work described in this paper was supported by Epigenomics, AG. The authors state no other conflict of interest.

1. Takai $\mathrm{D}$, Jones PA. Comprehensive analysis of $\mathrm{CpG}$ islands in human chromosomes 21 and 22. Proc Natl Acad Sci USA 2002;99:3740-3745.

2. Costello JF, Fruhwald MC, Smiraglia DJ, et al. Aberrant CpG-island methylation has non-random and tumour-type-specific patterns. Nat Genet 2000;24:132-138.

3. Esteller M, Corn PG, Baylin SB, et al. A gene hypermethylation profile of human cancer. Cancer Res 2001;61:3225-3229.

4. Eads CA, Lord RV, Wickramasinghe $\mathrm{K}$, et al. Epigenetic patterns in the progression of esophageal adenocarcinoma. Cancer Res 2001;61: 3410-3418.

5. Soria JC, Rodriguez M, Liu DD, et al. Aberrant promoter methylation of multiple genes in bronchial brush samples from former cigarette smokers. Cancer Res 2002;62:351-355.

6. Parkin DM, Bray F, Ferlay J, et al. Global cancer statistics, 2002. CA Cancer J Clin 2005;55:74-108.

7. Esteller M. CpG island hypermethylation and tumor suppressor genes: a booming present, a brighter future. Oncogene 2002;21:5427-5440.

8. Yamashita S, Tsujino Y, Moriguchi K, et al. Chemical genomic screening for methylation-silenced genes in gastric cancer cell lines using 5-aza$2^{\prime}$-deoxycytidine treatment and oligonucleotide microarray. Cancer Sci 2006;97:64-71.

9. Larsen F, Gundersen G, Lopez R, et al. CpG islands as gene markers in the human genome. Genomics 1992;13:1095-1107.

10. Antequera F, Bird A. Number of $\mathrm{CpG}$ islands and genes in human and mouse. Proc Natl Acad Sci USA 1993;90:11995-11999.

11. Saxonov S, Berg P, Brutlag DL. A genome-wide analysis of CpG dinucleotides in the human genome distinguishes two distinct classes of promoters. Proc Natl Acad Sci USA 2006;103:1412-1417.

12. Herman JG, Graff JR, Myohanen S, et al. Methylation-specific PCR: a novel PCR assay for methylation status of CpG islands. Proc Natl Acad Sci USA 1996;93:9821-9826.

13. Eads CA, Danenberg KD, Kawakami K, et al. MethyLight: a highthroughput assay to measure DNA methylation. Nucleic Acids Res 2000;28:E32.

14. Ogino S, Kawasaki T, Brahmandam $M$, et al. Precision and performance characteristics of bisulfite conversion and real-time PCR (MethyLight) for quantitative DNA methylation analysis. J Mol Diagn 2006;8:209-217.

15. Widschwendter $M$, Siegmund $K D$, Müller $H M$, et al. Association of breast cancer DNA methylation profiles with hormone receptor status and response to Tamoxifen. Cancer Res 2004;64:3807-3813.

16. Weisenberger DJ, Campan M, Long $\mathrm{Tl}$, et al. Analysis of repetitive element DNA methylation by MethyLight. Nucleic Acids Res 2005;33:6823-6836.

17. Ogino S, Cantor M, Kawasaki T, et al. CpG island methylator phenotype (CIMP) of colorectal cancer is best characterised by quantitative DNA methylation analysis and prospective cohort studies. Gut 2006;55: 1000-1006.

18. de Jong D, van der Hulst RW, Pals G, et al. Gastric non-Hodgkin lymphomas of mucosa-associated lymphoid tissue are not associated with more aggressive Helicobacter pylori strains as identified by CagA. Am J Clin Pathol 1996;106:670-675.

19. Lauren P. The two histologic main types of gastric carcinoma: diffuse and so-called intestinal-type carcinoma. Acta Pathol Microbiol Scand 1965;64:31-49.

20. Toyota $M$, Ahuja $N$, Ohe-Toyota $M$, et al. CpG island methylator phenotype in colorectal cancer. Proc Natl Acad Sci USA 1999;96: 8681-8686.
21. Kang GH, Lee S, Kim WH, et al. Epstein-Barr virus-positive gastric carcinoma demonstrates frequent aberrant methylation of multiple genes and constitutes $\mathrm{CpG}$ island methylator phenotype-positive gastric carcinoma. Am J Pathol 2002;160:787-794.

22. Chong JM, Sakuma K, Sudo M, et al. Global and non-random CpGisland methylation in gastric carcinoma associated with Epstein-Barr virus. Cancer Sci 2003;94:76-80.

23. An C, Choi IS, Yao JC, et al. Prognostic significance of CpG island methylator phenotype and microsatellite instability in gastric carcinoma. Clin Cancer Res 2005;11:656-663.

24. Widschwendter $M$, Fiegl $H$, Egle $D$, et al. Epigenetic stem cell signature in cancer. Nat Genet 2007;39:157-158.

25. Chan AO, Lam SK, Wong BC, et al. Promoter methylation of E-cadherin gene in gastric mucosa associated with Helicobacter pylori infection and in gastric cancer. Gut 2003;52:502-506.

26. Leung WK, Man EP, Yu J, et al. Effects of Helicobacter pylori eradication on methylation status of E-cadherin gene in noncancerous stomach. Clin Cancer Res 2006;12:3216-3221.

27. Maekita T, Nakazawa K, Mihara $M$, et al. High levels of aberrant DNA methylation in Helicobacter pylori-infected gastric mucosae and its possible association with gastric cancer risk. Clin Cancer Res 2006;12:989-995.

28. Waki T, Tamura G, Tsuchiya T, et al. Promoter methylation status of E-cadherin, hMLH1, and p16 genes in nonneoplastic gastric epithelia. Am J Pathol 2002;161:399-403.

29. Nakajima T, Maekita T, Oda I, et al. Higher methylation levels in gastric mucosae significantly correlate with higher risk of gastric cancers. Cancer Epidemiol Biomarkers Prev 2006;15:2317-2321.

30. Toyota $\mathrm{M}$, Ahuja $\mathrm{N}$, Suzuki $\mathrm{H}$, et al. Aberrant methylation in gastric cancer associated with the $\mathrm{CpG}$ island methylator phenotype. Cancer Res 1999;59:5438-5442.

31. Toyota M, Ho C, Ohe-Toyota $M$, et al. Inactivation of CACNA1G, a T-type calcium channel gene, by aberrant methylation of its $5^{\prime} \mathrm{CpG}$ island in human tumors. Cancer Res 1999;59:4535-4541.

32. Chang MS, Uozaki $\mathrm{H}$, Chong JM, et al. CpG island methylation status in gastric carcinoma with and without infection of Epstein-Barr virus. Clin Cancer Res 2006;12:2995-3002.

33. Kusano M, Toyota M, Suzuki $H$, et al. Genetic, epigenetic, and clinicopathologic features of gastric carcinomas with the CpG island methylator phenotype and an association with Epstein-Barr virus. Cancer 2006;106:1467-1479.

34. Ushiku T, Chong JM, Uozaki $\mathrm{H}$, et al. p73 gene promoter methylation in Epstein-Barr virus-associated gastric carcinoma. Int J Cancer 2007;120:60-66.

35. Tahara E, Semba S, Tahara H. Molecular biological observations in gastric cancer. Semin Oncol 1996;23:307-315.

36. Stadtlander CT, Waterbor JW. Molecular epidemiology, pathogenesis and prevention of gastric cancer. Carcinogenesis 1999;20: 2195-2208.

37. Chan AO, Luk JM, Hui WM, et al. Molecular biology of gastric carcinoma: from laboratory to bedside. J Gastroenterol Hepatol 1999;14:150-160.

38. Kaneda A, Kaminishi M, Yanagihara $K$, et al. Identification of silencing of nine genes in human gastric cancers. Cancer Res 2002;62: 6645-6650.

39. Oue $\mathrm{N}$, Oshimo $\mathrm{Y}$, Nakayama $\mathrm{H}$, et al. DNA methylation of multiple genes in gastric carcinoma: association with histological type and CpG island methylator phenotype. Cancer Sci 2003;94: 901-905.

40. Oue N, Mitani Y, Motoshita J, et al. Accumulation of DNA methylation is associated with tumor stage in gastric cancer. Cancer 2006;106:1250-1259. 